\title{
A VOLTERRA EQUATION WITH SQUARE INTEGRABLE SOLUTION
}

\author{
OLOF J. STAFFANS
}

\begin{abstract}
We study the asymptotic behavior of the solutions of the nonlinear Volterra integrodifferential equation

$$
x^{\prime}(t)+\int_{0}^{t} a(t-s) g(x(s)) d s=f(t) \quad\left(t \in R^{+}\right) .
$$

Here $R^{+}=[0, \infty), a, g$ and $f$ are given real functions, and $x$ is the unknown solution. In particular, we give sufficient conditions which imply that $x$ and $x^{\prime}$ are square integrable.
\end{abstract}

1. Introduction and summary of results. We study the asymptotic behavior of the solutions of the Volterra integrodifferential equation

$$
x^{\prime}(t)+\int_{0}^{t} a(t-s) g(x(s)) d s=f(t) \quad\left(t \in R^{+}\right) .
$$

Here $R^{+}=[0, \infty)$, the prime denotes differentiation, $a, g$ and $f$ are given, real functions, and $x$ is the unknown solution. In particular, we give sufficient conditions which imply that the solutions satisfy $x, x^{\prime} \in L^{2}\left(R^{+}\right)$.

Our assumptions are the following:

$$
a=b+c \text { is strongly positive definite, }
$$

where

$$
\begin{aligned}
& \quad b \in L^{1}\left(R^{+}\right) \text {satisfies }|\hat{b}(\omega)|^{2} \leqslant \beta \operatorname{Re} \hat{b}(\omega) \quad(\omega \in R) \\
& \text { for some } \beta \geqslant 0, \\
& \quad c \text { is positive definite, and } c^{\prime} \in L^{1} \cap \operatorname{BV}\left(R^{+}\right), \\
& g \in C(R), \xi g(\xi)>0 \quad(\xi \neq 0), \text { and } \liminf _{\xi \rightarrow 0} g(\xi) / \xi>0, \\
& f=f_{1}+f_{2}+f_{3}, \text { where } f_{1} \in L^{2}\left(R^{+}\right), f_{2} \in \mathrm{BV}\left(R^{+}\right), \\
& \text {and } f_{3} \in L^{\infty}\left(R^{+}\right), f_{3}^{\prime} \in L^{2}\left(R^{+}\right) .
\end{aligned}
$$

Here $\hat{b}(\omega)=\int_{0}^{\infty} e^{-i \omega t} b(t) d t$ is the Fourier transform of $b$. The strong positive definiteness of a means that there exists $\epsilon>0$ such that the function $a(t)-\epsilon e^{-t}$ is positive definite. The statements concerning $c^{\prime}$ and $f_{3}^{\prime}$ should be interpreted as requirements that $c, f_{3}$ be locally absolutely continuous, together with the additional conditions on the derivatives. BV stands for functions of bounded variation.

We call $x$ a solution of (1.1) if $x$ is locally absolutely continuous, and (1.1) holds a.e. In addition to (1.2)-(1.6) above we shall have to assume that a solution $x$ of

Received by the editors January 30, 1979.

AMS (MOS) subject classifications (1970). Primary 45D05, 45G99, 45J05, 45M05. 
(1.1) satisfies

$$
x, Q_{a} \in L^{\infty}\left(R^{+}\right)
$$

where

$$
Q_{a}(T)=\int_{0}^{T} g(x(t)) \int_{0}^{t} a(t-s) g(x(s)) d s d t \quad\left(T \in R^{+}\right) .
$$

Sufficient conditions for (1.7) to hold can be found in [7]. For example, any one of (1.9)-(1.11) below combined with (1.2)-(1.5) and the assumption

$$
-\int_{-\infty}^{0} g(\xi) d \xi=\int_{0}^{\infty} g(\xi) d \xi=\infty
$$

imply (1.7):

$$
\begin{gathered}
f \in L^{1}\left(R^{+}\right), \text {and } \limsup _{|\xi| \rightarrow \infty}|g(\xi)|\left(1+\int_{0}^{\xi} g(\eta) d \eta\right)^{-1}<\infty, \\
f, f^{\prime} \in L^{2}\left(R^{+}\right) \\
c(\infty)>0, \text { and } f \in \mathrm{BV}\left(R^{+}\right) .
\end{gathered}
$$

We prove the following result:

THEOREM 1. Let (1.2)-(1.6) hold, and let $x$ be a solution of (1.1) satisfying (1.7). Then $x, x^{\prime} \in L^{2}\left(R^{+}\right)$.

Theorem 1 is an improved version of [8, Theorem 1(iii)]. One gets [8, Theorem 1(iii)] by adding (1.10) and

$$
b \equiv 0, \quad c-c(\infty) \in L^{1}\left(R^{+}\right)
$$

to the hypothesis of Theorem 1.

Theorem 1 extends some of the results in [5] and [6]. The hypothesis used here is comparatively strong, but, on the other hand, we now get the stronger conclusion $x \in L^{2}\left(R^{+}\right.$) (which amounts to a faster convergence of $x$ to zero than [5] and [6] yield).

This work may be regarded as a strengthening of [8], which in turn was inspired by some estimates in the two papers [1] and [2] of MacCamy. In spite of this fact our argument is quite different from MacCamy's. MacCamy does not work with a scalar equation as we do, but with an abstract Volterra equation of hyperbolic type. We shall return elsewhere [10] to the question of how the estimates in the proof of Theorem 1 should be modified in the abstract case.

We discuss conditions (1.2)-(1.4) in $\$ 3$.

\section{Proof of Theorem 1. Define}

$$
\varphi(t)=g(x(t)), \quad d(t)=(1+c(0)) e^{-t} \quad\left(t \in R^{+}\right) .
$$

Let * denote convolution, subtract $d * \varphi$ from both sides of (1.1), and use (1.2), (1.6) to get

$$
x^{\prime}-(d-c) * \varphi-f_{2}-f_{3}=f_{1}-(b+d) * \varphi
$$

Define

$$
u=(d-c) * \varphi, \quad v=(d-c)^{\prime} * \varphi, \quad w=(b+d) * \varphi
$$


Multiply (2.1) by $x^{\prime}$, integrate over $(0, T)$, and integrate the terms on the left-hand side by parts (except the first one) to get

$$
\begin{aligned}
\int_{0}^{T}\left[x^{\prime}(t)\right]^{2} d t & +\int_{0}^{T} x(t) g(x(t)) d t \\
= & x(T)\left[u(T)+f_{2}(T)+f_{3}(T)\right]-x(0) f_{3}(0) \\
& -\int_{0}^{T} x(t) d f_{2}(t)+\int_{0}^{T} x^{\prime}(t)\left[f_{1}(t)-w(t)\right] d t \\
& -\int_{0}^{T} x(t)\left[v(t)+f_{3}^{\prime}(t)\right] d t
\end{aligned}
$$

where we have redefined $f_{2}$ so that it is continuous from the left, and $f_{2}(0)=0$. By the Hölder and Minkowski inequalities,

$$
\begin{gathered}
\left\|x^{\prime}\right\|_{2}^{2}+\int_{0}^{T} x(t) g(x(t)) d t \leqslant\left(\|u\|_{\infty}+\left\|f_{2}\right\|_{\infty}+\left\|f_{2}\right\|\|+2\| f_{3} \|_{\infty}\right)\|x\|_{\infty} \\
+\left(\left\|f_{1}\right\|_{2}+\|w\|_{2}\right)\left\|x^{\prime}\right\|_{2}+\left(\|v\|_{2}+\left\|f_{3}^{\prime}\right\|_{2}\right)\|x\|_{2},
\end{gathered}
$$

where \|\|$_{p}(p=2, \infty)$ is the norm of $L^{p}(0, T)$, and $\left\|\mid f_{2}\right\|$ is the total variation of $f_{2}$.

We claim that

$$
u \in L^{\infty}\left(R^{+}\right), \quad v, w \in L^{2}\left(R^{+}\right) .
$$

Assume this for the moment. Then, by (1.6), (1.7) and (2.4), inequality (2.3) is of the form

$$
\left\|x^{\prime}\right\|_{2}^{2}+\int_{0}^{T} x(t) g(x(t)) d t \leqslant C\left(1+\left\|x^{\prime}\right\|_{2}+\|x\|_{2}\right),
$$

where $C$ is a (sufficiently large) constant independent of $T$. Observe that (1.5), (1.7) imply the existence of $\epsilon>0$ such that $x(t) g(x(t)) \geqslant \epsilon|x(t)|^{2}\left(t \in R^{+}\right)$. Hence (2.5) becomes

$$
\left\|x^{\prime}\right\|_{2}^{2}+\epsilon\|x\|_{2}^{2} \leqslant C\left(1+\left\|x^{\prime}\right\|_{2}+\|x\|_{2}\right),
$$

from which the conclusion of Theorem 1 follows.

It remains to verify the crucial estimate (2.4). Observe that the functions $b, c$ and $d$ are all positive definite, and that by (1.2), $0 \leqslant Q_{b}(T) \leqslant Q_{a}(T), 0 \leqslant Q_{c}(T) \leqslant$ $Q_{a}(T)$, and $0 \leqslant Q_{d}(t) \leqslant C Q_{a}(T)$, where $Q_{b}, Q_{c}$ and $Q_{d}$ are defined as in (1.8), and $C$ is some positive constant. Thus (1.7) implies

$$
Q_{b}, Q_{c}, Q_{d} \in L^{\infty}\left(R^{+}\right) \text {. }
$$

Both $c$ and $d$ are continuous and positive definite, and so [4, Lemma 6.1] yields

$$
|c * \varphi(T)|^{2} \leqslant 2 c(0) Q_{c}(T), \quad|d * \varphi(T)|^{2} \leqslant 2 d(0) Q_{d}(T) .
$$

Combining (2.2) with (2.6) and (2.7) one gets the first part of (2.4). By (1.3) and [5, Lemma 1],

$$
\|b * \varphi\|_{2}^{2} \leqslant \beta Q_{b}(T) .
$$

Observe that $c^{\prime}, d, d^{\prime} \in L^{1} \cap \mathrm{BV}\left(R^{+}\right)$, and use (1.2) and [9, Lemma 2.2] to get

$$
\left\|c^{\prime} * \varphi\right\|_{2}^{2}+\|d * \varphi\|_{2}^{2}+\left\|d^{\prime} * \varphi\right\|_{2}^{2} \leqslant C Q_{a}(T)
$$


for some constant $C$. Combining this with (1.7), (2.2), (2.6) and (2.8) we get the second part of (2.4). This completes the proof of Theorem 1.

3. Comments. The proof of Theorem 1 gives us, in fact, a little more than $x \in L^{2}\left(R^{+}\right)$, namely

$$
\int_{0}^{\infty} x(t) g(x(t)) d t<\infty
$$

(cf. (2.5)). If $\lim \sup _{\xi \rightarrow 0} g(\xi) / \xi<\infty$, then (3.1) is equivalent to $x \in L^{2}\left(R^{+}\right)$. However, if e.g., $g(\xi)=\xi^{1 / 3}$ (which satisfies (1.5)), then (3.1) becomes $x \in$ $L^{4 / 3}\left(R^{+}\right)$.

The conditions (1.2)-(1.4) require a splitting of $a$ into two parts, and given $a$ it is not always obvious how this splitting should be done. Some requirements are obvious: If $a$ or $a^{\prime}$ is unbounded, then the unbounded part must go into $b$, and if $a$ is not integrable, then the nonintegrable part must go into $c$. Below we shall give some examples where the splitting succeeds. For example, in the following two cases (1.3) holds:

$$
\begin{gathered}
b \in L^{1} \cap \mathrm{BV}\left(R^{+}\right) \text {is strongly positive definite, } \\
b \in L^{1}\left(R^{+}\right), \text {and } b,-b^{\prime} \text { are convex }
\end{gathered}
$$

(see [5, Theorem 2]). Thus, if, e.g., $a$ is strongly positive definite, and $a-a(\infty) \in$ $L^{1} \cap \mathrm{BV}\left(R^{+}\right)$, then one can take $b=a-a(\infty), c=a(\infty)$ (the strong positive definiteness of $a$ implies the strong positive definiteness of $b$ in this case, and $a(\infty)>0)$. On the other hand, if $a^{\prime} \in L^{1} \cap \mathrm{BV}\left(R^{+}\right)$, then one may choose $b \equiv 0$, $c=a$. An example where (3.3) is used is the following: Suppose that $a(t)=t^{-\alpha}$ $\left(t \in R^{+}\right)$, where $0<\alpha<1$, and define $b(t)=t^{-\alpha}-(1+t)^{-\alpha}, c(t)=(1+t)^{-\alpha}$ (cf. [3, Corollary 2.2]).

One way of simplifying the problem of how one should split $a$ into $b+c$ is to modify (1.3), (1.4), and modify the proof of Theorem 1 accordingly. One can replace (1.3), (1.4) by

$$
\begin{aligned}
& b \in L^{1}\left(R^{+}\right) \text {, and }|\hat{b}(\omega)|^{2} \leqslant \beta \operatorname{Re} \hat{a}(\omega)(\omega \in R) \\
& \text { for some } \beta>0, \\
& \qquad \quad c \in L^{2}\left(R^{+}\right), \quad c^{\prime} \in L^{1} \cap \operatorname{BV}\left(R^{+}\right) .
\end{aligned}
$$

Most of the proof of Theorem 1 remains valid. (2.6) should be replaced by

$$
Q_{e} \in L^{\infty}\left(R^{+}\right)
$$

where $e(t)=e^{-t}\left(t \in R^{+}\right)$, and (2.7), (2.8) by

$$
\begin{aligned}
|c * \varphi(T)|^{2} \leqslant & 2 Q_{e}(T) \int_{0}^{\infty}\left(c^{2}(t)+\left[c^{\prime}(t)\right]^{2}\right) d t, \\
|d * \varphi(T)|^{2} \leqslant & 2(1+c(0))^{2} Q_{e}(T), \\
& \|b * \varphi\|_{2}^{2} \leqslant \beta Q_{a}(T) .
\end{aligned}
$$

The proofs of (3.6), (3.7) are similar to the proofs of [4, Lemma 6.1] and [5, Lemma 1].

In (3.4), (3.5) it is no longer required that $b$ and $c$ be positive definite, which 
clearly facilitates the splitting of $a$ into $b+c$. In particular, (3.2) can be weakened to $b \in L^{1} \cap \mathrm{BV}\left(R^{+}\right)$. On the other hand, the added condition $c \in L^{2}\left(R^{+}\right)$ prevents the use of (3.4), (3.5), e.g., when $a(t)=t^{-\alpha}$ with $0<\alpha<\frac{1}{2}$. Also observe that (3.4), (3.5) exclude the possibility $a(\infty)>0$.

\section{REFERENCES}

1. R. C. MacCamy, An integro-differential equation with application in heat flow, Quart. Appl. Math. 35 (1977), 1-19.

2. __ A model for one-dimensional, nonlinear viscoelasticity, Quart. Appl. Math. 35 (1977), 21-33.

3. J. A. Nohel and D. F. Shea, Frequency domain methods for Volterra equations, Advances in Math. 22 (1976), 278-304.

4. O. J. Staffans, Positive definite measures with applications to a Volterra equation, Trans. Amer. Math. Soc. 218 (1976), 219-237.

5. __ An inequality for positive definite Volterra kernels, Proc. Amer. Math. Soc. 58 (1976), 205-210.

6. On the asymptotic spectra of the bounded solutions of a nonlinear Volterra equation, J. Differential Equations 24 (1977), 365-382.

7. _ Boundedness and asymptotic behavior of solutions of a Volterra equation, Michigan Math. J. 24 (1977), 77-95.

8. A nonlinear Volterra integral equation with square integrable solutions, Volterra Equations, S.-O. Londen and O. J. Staffans, Eds., Lecture Notes in Math., vol. 737, Springer-Verlag, Berlin, 1979, pp. 281-286.

9. , A nonlinear Volterra equation with rapidly decaying solutions, Trans. Amer. Math. Soc. (to appear).

10. __ On a nonlinear hyperbolic Volterra equation, SIAM J. Math. Anal. (to appear).

Institute of Mathematics, HeLSinki University of Technology, SF-02150 EsPOO 15, FinLAND 